\title{
Carbon-10: Example of cyclotron production of positron emitters as an open research field
}

\author{
F. Alves ${ }^{\mathrm{a}, *}$, J.J.P. Lima ${ }^{\mathrm{b}}$, R.J. Nickles ${ }^{\mathrm{c}}$, M. Jensen ${ }^{\mathrm{d}}$ \\ ${ }^{a}$ Escola Superior de Tecnologia da Saúde de Coimbra, Avenida 5 de Outubro, P-3046 Coimbra, Portugal \\ b IBILI, Faculdade de Medicina da Universidade de Coimbra, P-3000 Coimbra, Portugal \\ ${ }^{\mathrm{c}}$ Medical Physics Department, 1530 Medical Science Center, 1300 University Avenue, University of Wisconsin, Madison, \\ WI 53706 1532, USA \\ ${ }^{\mathrm{d}}$ Risø National Laboratory, DK-4000 Roskilde, Denmark
}

\begin{abstract}
This paper supports the thesis that significant improvement of PET output response to clinical questions can be achieved by innovation in radionuclide production. Moreover, that development can be performed with the resources available at a clinical centre. Carbon-10 production parameters studies are used as example. A technical methodology for measurement of excitation function of nuclear reactions yielding short-lived radionuclides, performed to measure cross section values of the ${ }^{10} \mathrm{~B}(\mathrm{p}, \mathrm{n}){ }^{10} \mathrm{C}$ reaction in a PET-devoted cyclotron, is presented.
\end{abstract}

(C) 2006 Elsevier Ltd. All rights reserved.

\section{Introduction}

Positron emission tomography (PET) is a quantitative functional imaging technique with numerous applications in both clinical practice and research. The high sensitivity derived from the use of radioisotopic methods, together with the availability of positron-emitting isotopes of the most prevailing atoms in the organic molecules, confers to PET a unique role within the noninvasive molecular imaging techniques for studies in man (Jones, 1996).

A PET study can be, synthetically, divided into a set of sequential tasks: production of the positron-emitting nuclides, radiosynthesis and labelling, radiation emission and detection, image processing and analysis. Although all steps are important for the quality and relevance of the final result, a concentration of methodological innovation

\footnotetext{
*Corresponding author. Fax: + 351239813395.

E-mail address: falves@estescoimbra.pt (F. Alves).
}

and development in the post-acquisition and image quantification processes has been observed in the past few years. This trend cannot be detached from the availability of established radiotracers for quantification of several clinically important processes (e.g., fluor-18labelled fluorodeoxyglucose for the oxidative metabolism and oxygen-15-labelled water for regional cerebral blood flow), decisive for the success of PET and the widespread proliferation of small dedicated clinical centres. This scenario contributes to generalise the idea that there is not much more to be developed in the basic areas (radionuclide production and radiochemistry), deemed as needing "large resources".

We hereby make an effort to invert this trend, showing that methodological and technical innovation involving research in radionuclide production can lead to the improvement of PET output response to clinical questions. Moreover, this development can be performed within a clinically oriented PET centre with a low/medium energy cyclotron. 


\section{Cyclotron radionuclide production; excitation function of nuclear reactions}

Radionuclide production in a cyclotron is based upon target material nuclide transmutation. The leading physical process, a nuclear reaction induced by cyclotron beam irradiation, can be quantified by its excitation function, which plots the reaction cross section (measure of the physical probability of occurrence) as function of the incoming beam energy.

Unknown excitation functions are usually determined within the clinically oriented cyclotron centre by an experimental method commonly called "stacked foil" (Qaim, 1982). This traditional protocol consists of a single irradiation of a stack of foils, followed by sequential measurement of the activity induced in each foil. It is assumed that the decay rate of the nuclear reaction product is small when compared with the time required to sequentially measure the activity in the foils ensuring adequate counting statistics. Therefore, the decay constant is a limiting factor for the number of foils that can be measured, bearing in mind that the possibility of simultaneously measuring each foil in a different detector is not cost-effective.

\section{Proposed methodology for measurement of excitation functions of nuclear reactions yielding short-lived radionuclides}

In order to measure excitation functions of nuclear reactions yielding short-lived products, an alternative methodology is proposed. Instead of a single irradiation, the proposed method uses several irradiations of stacks where only one of the foils is under study. Each irradiation is followed by activity measurement. The remaining foils in the stacks, of calibrated thickness and adequate composition, determine the effective energy of the beam in the foil under study. The proper use of this methodology implies that all cyclotron parameters remain constant during irradiations and in-between different irradiations. However, in the common cyclotron operating protocols, obtaining a determined value for the beam current is an interactive process between operator settings and ion beam response. Such a process is hardly compatible with the proposed method, forcing a reprogramming of the cyclotron beam production sequence. The main beam switch will operate the RF system, instead of the ion source. The ion source parameters are set and stabilized before the irradiation protocol, not requiring further adjustment by the cyclotron operator. This way, the source will be producing, at constant rate, ions that, when the RF is switched on, are just accelerated, resulting in a constant current beam. Practical application of the proposed methodology, including the cyclotron-modified proto- col, leads to results with precision comparable to the one achieved with the conventional "stacked-foil" method.

\section{Cyclotron production of positron emitters for medical use: physical/chemical constrains}

The excitation functions of the different nuclear reactions that can produce a certain nuclide, in the experimental conditions (i.e., available projectiles and respective energy), determine the hierarchy of these reactions in terms of production efficiency. However, the process of establishing a production protocol for a certain radioisotope is not restricted to the choice of the nuclear reaction that leads to the largest production yield of that isotope. It also requires further evaluation of the candidate reactions, bearing in mind the physical/ chemical characteristics, commercial availability and price of the target material, and the viability, yield and timing of the extraction/separation process of the nuclide under production. Moreover, the relation between a given projectile-target pair and a certain nuclear reaction is not necessarily univocal, even for a certain beam energy value. Thus, other competing nuclear reactions, leading to impurities in the final product, have often to be taken into account. Eventually, the impossibility of keeping low levels of impurities may preclude the use of the production protocol based in a certain nuclear reaction. When these impurities are not radioisotopes of the intended nuclide, they can be separated, referring to the physical/chemical characterization of the target. If they correspond to radioisotopes of the intended nuclide, a compromise, in terms of maximising production and minimising impurities, can be achieved using an appropriate projectile energy range in the target. This optimisation process implies the knowledge of the beam energy degradation in the target material, reflecting the correspondent stopping power. The stopping power of most elements for the light projectiles typically accelerated in cyclotrons, in a large range of beam energy values, is readily available from literature (Williamson et al., 1966) and/or can easily be calculated within acceptable error tolerance from algorithms based on Bethe's theoretical expression (Bethe, 1930).

\section{A clinical question answered by a new positron emitter, carbon-10 $\left({ }^{10} \mathrm{C}\right)$}

A continuous tracer administration method (Jones et al., 1976), based on Kety's single compartment model (Kety, 1951), has been used since the 1980s for mapping regional cerebral blood flow (rCBF) using PET (Frackowiak et al., 1980). Using oxygen-15 (as tracer in $\mathrm{H}_{2}^{15} \mathrm{O}$, $\mathrm{C}^{15} \mathrm{O}_{2}$ or $\mathrm{C}^{15} \mathrm{O}$ ), this steady-state imaging methodology 
is intrinsically limited by a non-linearity in the local blood flow to amount of tracer ratio, due to the relatively slow decay rate of the positron emitter $\left(\lambda=0.34 \mathrm{~min}^{-1}\right)$ (Huang et al., 1979).

Neon-19 $\left(\mathrm{Ne}^{19}, \lambda=2.4 \mathrm{~min}^{-1}\right)$ would overcome this effect (Kearfott et al., 1983), but has low blood solubility.

Nowadays, the method of choice for rCBF mapping with PET is a variation of the steady-state methodology, based on a $\mathrm{H}_{2}^{15} \mathrm{O}$ bolus injection (Huang et al., 1982), imposing anyway a typical interval of $10 \mathrm{~min}$ between repeated studies.

In this context, facing a clinical question demanding activation studies with time intervals down to 2 min between changes in stimuli, the established methodology is unable to provide an answer.

A qualified reply to the clinical question can be achieved by the introduction of ${ }^{10} \mathrm{C}$-labelled carbon dioxide $\left(\left[{ }^{10} \mathrm{C}\right] \mathrm{CO}_{2}\right)$ as a new tracer for mapping regional cerebral blood flow with PET (Jensen et al., 1998; Nickles et al., 1998). ${ }^{10} \mathrm{C}$ decay rate $\left(\lambda=2.2 \mathrm{~min}^{-1}\right)$, fast when compared to its transport kinetics, and $\mathrm{CO}_{2}$ good solubility in blood and tissue, allows steady-state flow imaging using single-frame static data acquisitions during continuous tracer administration, with negligible intrinsic methodological limitations (Jensen et al., 1999).

\section{Cyclotron production of ${ }^{10} \mathrm{C}$}

The potential for widespread use of this tracer depends on the possibilities of ${ }^{10} \mathrm{C}$ production in PET dedicated cyclotrons. It should also be taken in mind that the viability of continuous steady-state administration of $\left[{ }^{10} \mathrm{C}_{\mathrm{CO}_{2}}\right.$ imposes that longer-lived radionuclide impurities do not build up during production and depends on a reliable on-line chemistry system.

The most plausible choice for ${ }^{10} \mathrm{C}$ production is the ${ }^{10} \mathrm{~B}(\mathrm{p}, \mathrm{n}){ }^{10} \mathrm{C}$ reaction. Since monoisotopic ${ }^{10} \mathrm{~B}$ is unavailable, the simultaneous production of ${ }^{11} \mathrm{C}$ is unavoidable through the ${ }^{11} \mathrm{~B}(\mathrm{p}, \mathrm{n}){ }^{11} \mathrm{C}$.

The previously unknown excitation function for the reaction ${ }^{10} \mathrm{~B}(\mathrm{p}, \mathrm{n}){ }^{10} \mathrm{C}$ was experimentally determined (Alves et al., 2000) using the proposed methodology for measurement in the situation of nuclear reactions yielding short-lived products. The results obtained were used, together with data for the ${ }^{11} \mathrm{~B}(\mathrm{p}, \mathrm{n}){ }^{11} \mathrm{C}$ reaction, available from the literature (Firouzbakht et al., 1998) to optimise production parameters, namely proton beam energy and target thickness, in order to maximise ${ }^{10} \mathrm{C}$ to ${ }^{11} \mathrm{C}$ ratio while maintaining high ${ }^{10} \mathrm{C}$ production yields.

For commercially available isotopic enrichments, in saturation condition for both isotopes, the level of the ${ }^{11} \mathrm{C}$ impurity is $12 \%$ for $11 \mathrm{MeV}$ proton beam production. This level decreases for higher beam energy values as a result from a more favourable ratio between the

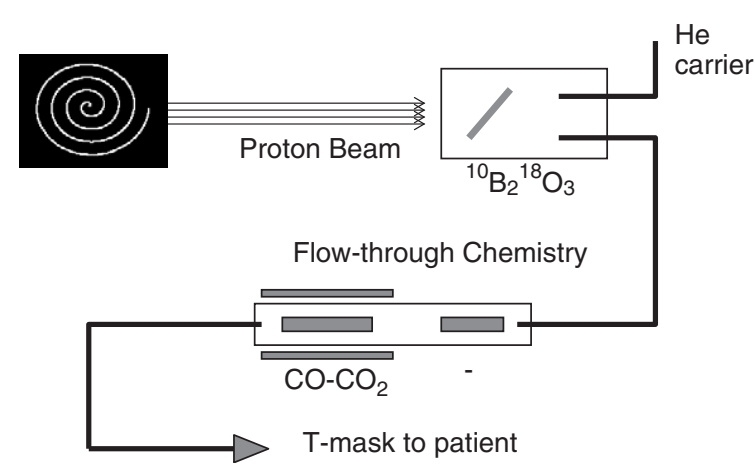

Fig. 1. On-line $\left[{ }^{10} \mathrm{C}\right] \mathrm{CO}_{2}$ from cyclotron-produced ${ }^{10} \mathrm{C}$.

cross sections. The optimum determined energy range (Alves et al., 2000) corresponds to a beam degradation from 28 to $14 \mathrm{MeV}$, corresponding to an isotopic impurity level of $3.2 \%$.

In terms of thick target yield, in a $11 \mathrm{MeV}$ cyclotron about $450 \mathrm{MBq}$ of ${ }^{10} \mathrm{C}$ can be produced, per $\mu \mathrm{A}$ in saturation, using the ${ }^{10} \mathrm{~B}(\mathrm{p}, \mathrm{n}){ }^{10} \mathrm{C}$ reaction. The same reaction yields more than $1800 \mathrm{MBq}$ per $\mu \mathrm{A}$, in saturation, using an $18 \mathrm{MeV}$ cyclotron.

For practical production (Jensen et al., 1998; Nickles et al., 1998), molten ${ }^{10} \mathrm{~B}_{2}^{18} \mathrm{O}_{3}$ on a rhenium mesh support can be used. The target material enrichment should be highest possible to reduce ${ }^{11} \mathrm{C}$ and ${ }^{13} \mathrm{~N}$ production. The primary product is ${ }^{10} \mathrm{CO}$, which is swept from the target in a tuned helium flow to a furnace system based on $\mathrm{CuO}$, yielding pure ${ }^{10} \mathrm{CO}_{2}$, which can be administered through a normal T-piece breathing mask (Fig. 1).

The system of Fig. 1 was implemented within a hospital PET centre with cyclotron, the Cyclotron and PET Center of Rigshospitalet, Denmark's National Hospital in Copenhagen. All research and development work, proving that methodological and technical innovation is possible in one of the most fundamental components of the PET technique - radionuclide production, was entirely performed in this clinically oriented centre.

\section{References}

Alves, F., Jensen, M., Jensen, H.J., Nickles, R.J., Holm, S., 2000. Determination of the excitation function for the ${ }^{10} \mathrm{~B}(\mathrm{p}, \mathrm{n}){ }^{10} \mathrm{C}$ reaction with implications for the production of $\left[{ }^{10} \mathrm{C}\right]$ carbon dioxide for use as a PET tracer. Appl. Radiat. Isot. 52, 899-903.

Bethe, H.A., 1930. Theory of the passage of fast corpuscular rays through matter. Ann. Physik 5, 325-400.

Firouzbakht, M.L., Schyler, D.J., Wolf, A.P., 1998. Yield measurements for the ${ }^{11} \mathrm{~B}(\mathrm{p}, \mathrm{n}){ }^{11} \mathrm{C}$ and the ${ }^{10} \mathrm{~B}(\mathrm{~d}, \mathrm{n}){ }^{11} \mathrm{C}$ nuclear reactions. Nucl. Med. Biol. 25, 161-164.

Frackowiak, R.S.J., Lenzi, G.-L., Jones, T., Heather, J.D., 1980. Quantitative measurement of regional cerebral blood 
flow in man, using oxygen-15 and positron emission tomography: theory procedure and normal values. J. Comput. Assist. Tomogr. 4, 727-736.

Huang, S.-C., Phelps, M.E., Hoffman, E.J., Kuhl, D.E., 1979. A theoretical study of quantitative flow measurements with constant infusion of short-lived isotopes. Phys. Med. Biol. 24, 1151-1161.

Huang, S.-C., Carson, R.E., Phelps, M.E., 1982. Measurement of local blood flow and distribution volume with short-lived isotopes: a general input technique. J. Cereb. Blood Flow Metabol. 2, 99-108.

Jensen, M., Nickles, R.J., Holm, S., 1998. Carbon-10 dioxide: a new tracer for human brain mapping with PET. Neuroimage 7, 630 .

Jensen, M., Holm, S., Nickles, R.J., Alves, F., 1999. Optimal use of ${ }^{10} \mathrm{CO}_{2}$ as a PET flow tracer in activation studies. J. Cereb. Blood Flow Metabol. 19, S813.

Jones, T., 1996. The role of positron emission tomography within the spectrum of medical imaging. Eur. J. Nucl. Med. 23, 207-211.
Jones, T., Chester, D.A., Ter-Pogossian, M.M., 1976. The continuous inhalation of oxygen-15 for assessing regional oxygen extraction in the brain of man. Br. J. Radiol. 49, 339-343.

Kearfott, K.J., Rottenberg, D.A., Volpe, B.T., 1983. Design of steady-state positron emission tomography protocols for neurobehavioral studies: $\mathrm{CO}^{15} \mathrm{O}$ and ${ }^{19} \mathrm{Ne}$. J. Comp. Assist. Tomogr. 7, 51-58.

Kety, S.S., 1951. The theory and applications of the exchange of inert gas at the lungs and tissues. Pharmacol. Rev. 3, $1-41$.

Nickles, R.J., Jensen, M., Holm, S., 1998. Carbon-10 dioxide: reviving steady state PET imaging of rCBF. J. Nucl. Med. 39 (Suppl. 5), 131.

Qaim, S.M., 1982. Nuclear data relevant to cyclotron produced short-lived medical radioisotopes. Radioch. Acta 30, 147-162.

Williamson, C.F., Boujot, J.P., Pickard, J., 1966. Tables of range and stopping power of chemical elements for charged particles of energy $0.05-500 \mathrm{MeV}$. Report CEA-R 3042. 